\title{
Assessing the validity of computer-game-like tests of processing speed and working memory
}

\author{
Jason MCPherson ANd Nicholas R. Burns \\ University of Adelaide, Adelaide, South Australia
}

\begin{abstract}
Processing speed (Gs) and working memory (WM) tasks have received considerable interest as correlates of more complex cognitive performance measures. Gs and WM tasks are often repetitive and are often rigidly presented, however. The effects of Gs and WM may, therefore, be confounded with those of motivation and anxiety. In an effort to address this problem, we assessed the concurrent and predictive validity of computer-game-like tests of Gs (Space Code) and WM (Space Matrix) across two experiments. In Experiment 1, within a university sample $(N=70)$, Space Matrix exhibited concurrent validity as a WM measure, whereas Space Code appeared to be a mixed-ability measure. In Experiment 2, Space Matrix exhibited concurrent validity as well as predictive validity (as a predictor of school grades) within a school-aged sample $(N=94)$, but the results for Space Code were less encouraging. Relationships between computer-game-like tests and gender, handedness, and computergame experience are also discussed.
\end{abstract}

One of the key challenges within research on cognitive abilities has been to identify simple cognitive tasks that can explain individual variation in more complex cognitiveperformance criteria such as composite ability (IQ) measures, fluid intelligence (Gf), ${ }^{1}$ and academic achievement (Deary, 2001; Engle, Tuholski, Laughlin, \& Conway, 1999; Jensen, 1998; McGrew, 2005; Schweizer, 2005). Most of the simple cognitive tasks that have been identified within this research tradition were originally developed as experimental measures of underlying "information-processing" components that were derived from various models of human cognition (see Floyd, 2005, for a review). Two informationprocessing components that have received considerable interest and support as correlates of more complex cognitiveperformance measures are processing speed and working memory (Conway, Cowan, Bunting, Therriault, \& Minkoff, 2002; Engle et al., 1999; Fry \& Hale, 1996; Luo, Thompson, \& Detterman, 2006; McGrew, 2005).

Processing speed (Gs) is measured using tasks that require rapid performance of simple cognitive operations. Gs tasks are so uniformly simple that people generally differ only in their speed of responding rather than in their accuracy (Carroll, 1993; Horn \& Noll, 1997). In contrast, working memory (WM) measures are primarily measures of accuracy, requiring the maintenance of "memory representations in the face of concurrent processing, distraction, and/or attention shifts" (Conway et al., 2002, p. 164). What Gs and WM tasks share are their relatively homogeneous measurement units: Gs is measured using the number of uniformly simple items completed within a given time limit; WM is measured using the number of uniformly simple representations maintained.
The precise relationship between Gs measures and more complex ability measures has been investigated and debated for decades (Burns \& Nettelbeck, 2003; Danthiir, Roberts, Schulze, \& Wilhelm, 2005; Jensen, 1982, 1998; Nyborg, 2003; Stankov \& Roberts, 1997). Similarly, a voluminous amount of research and commentary has focused on the relationship between WM and more complex ability measures (Ackerman, Beier, \& Boyle, 2005; Gathercole \& Pickering, 2000; Just \& Carpenter, 1992; Kane, Hambrick, \& Conway, 2005; Kyllonen \& Christal, 1990; Oberauer, Schulze, Wilhelm, \& Süß, 2005; Wickelgren, 1997). More recently, researchers have begun to investigate the complex interrelationships among Gs, WM, Gf, and broader composite ability measures (Conway et al., 2002; Fry \& Hale, 1996, 2000; McGrew, 2005; Schmid \& Leiman, 1957; Süß, Oberauer, Wittmann, Wilhelm, \& Schulze, 2002). The relatively consistent findings of substantial relationships among these variables has led some researchers to propose that Gs and WM tasks are viable assessment tools in educational and clinical settings (Lengenfelder et al., 2006; Luo et al., 2006).

Luo, Thompson, and Detterman (2006) argued that Gs and WM tasks require little formal instruction and do not seem to require the higher order processes thought to underlie complex reasoning. Additionally, the relatively homogeneous nature of items in Gs and WM tasks means that item banks can be generated easily and that they are amenable to experimental manipulation. This ease of manipulation also extends to the content and modality features of the stimuli used, so that Gs and WM tasks can be constructed using verbal, numerical, or spatial stimuli and can be presented visually or aurally in many cases.

J. McPherson, jlmcpher@psychology.adelaide.edu.au 
Although there is some evidence that domain-specific processes contribute to Gs and WM task performance, research has also suggested that the same underlying processes are measured at a broader level (McGrew, 2005; Miyake, 2001). Despite the aforementioned advantages and the finding that Gs and WM measures were predictors of scholastic achievement, however, Luo et al. also acknowledged that these tasks tend to be rigidly presented and often appear "impersonal." Consequently, they suggested that measurement of Gs and WM might be confounded to a greater extent with psychological effects such as anxiety and weakened motivation (p. 81).

We and other researchers have suggested that computergame-like tasks may provide a less anxiety-provoking and more motivating environment for those being tested (McPherson \& Burns, 2005, 2007; Porter, 1995; Washburn, 2003). This may be especially true for children, who are increasingly likely to be familiar with computers as a result of their experience with computer games (Gentile \& Walsh, 2002; Yelland \& Lloyd, 2001). Computer games incorporate a range of unique structural features that act to engage and motivate players, such as real-time positive and negative feedback (via graphics, sounds, and scoring), realistic graphics and sounds, and player advancement through levels (Wood, Griffiths, Chappell, \& Davies, 2004). With the incorporation of such features, computer-game-like Gs and WM tasks could potentially maintain many of the advantages inherent in such measures, while also addressing the concerns raised by Luo et al. (2006).

Alternatively, introducing game-like elements might distract those being tested to the point that the construct of interest is no longer being measured reliably. Indeed, since Gs and WM measures are also thought to involve strong attentional demands (Conway et al., 2002; Horn \& Blankson, 2005; Schweizer \& Moosbrugger, 2004)—and since such tests may also measure the ability to concentrate on somewhat monotonous tasks-altering motivationally sensitive aspects of the tasks could affect their psychometric properties. We believe, however, that the development of game-like tests may help researchers investigate the separate contributions of speed and attention in Gs tasks once their psychometric properties have been established. In our previous research, we have attempted to address this problem, as well as the additional ergonomic issue of using a computer mouse response method, by developing a computer-game-like test of Gs in distinct stages.

In a preliminary study, we developed a simple speeded coding task, using a mouse response method and nongame-like stimuli, and found that the task loaded on a Gs factor (McPherson \& Burns, 2005). In two subsequent studies, we investigated the impact of introducing gamelike features (McPherson \& Burns, 2007). In the first of these studies, we introduced complex game-like graphics as stimuli and found that the task shared variance with both Gs and visuospatial (Gv) tests. In the final study, we added further dynamic game-like features (e.g., scoring, feedback, and additional graphics and sound features) and assessed the task within a larger range of ability tests. The task, Space Code, was found to load strongly on a Gs factor and only minimally on a Gv factor. We concluded that
Space Code may be a viable measure of Gs, albeit one requiring further research, but there was also some evidence suggesting that the task might have a more complex psychometric profile.

The development of Space Code was partially inspired by the observation that many computer games inherently require rapid response of a nature similar to that of many Gs tasks. Our development of Space Code was also made easier by the fact that Gs tasks can be readily adapted to different stimulus classes, including game-like graphics in this instance. Similarly, it could also be argued that many modern games require the maintenance of memory representations in the face of other distracting tasks - much as WM tasks do - such as recalling where objects, doorways, or other rewards are located. Additionally, as with Space Code, there appears to be no reason why game-like graphics cannot be used as stimuli within a WM task.

The present article describes two related experiments. The overall aim of both experiments was to further assess the validity of Space Code as a measure of Gs, as well as to evaluate a new computer-game-like WM task, Space Matrix. Space Matrix was developed by using the core task of Space Code and adding a simultaneous task based on the dot matrix task developed by Miyake, Friedman, Rettinger, Shah, and Hegarty (2001). In Experiment 1, the concurrent validity of both tests was assessed in relation to established Gs, WM, and Gf measures, using a university student sample. In Experiment 2, concurrent validity was assessed as it was in Experiment 1, and predictive validity was assessed using school subject grades, both within a sample of school-aged children. Additionally, each experiment assessed relationships with gender, handedness, and computer-game experience measures.

\section{EXPERIMENT 1}

\section{Method}

\section{Participants}

There were 70 participants. All participants were Level I psychology students from the University of Adelaide who received course credit for their participation. The mean age for the whole sample was 19.6 years $(S D=4.00) ; 40$ were female (mean $=19.20$ years, $S D=$ $2.37)$, and 30 were male (mean $=20.13$ years, $S D=5.48)$.

\section{Materials}

Nonability measures. Participants were asked to indicate their age (in years), their gender, and whether they considered themselves predominantly right-handed, somewhat ambidextrous, or predominantly left-handed (scored 1, 2, and 3, respectively). Additionally, three questions were aimed at measuring computer game experience. The questions were (1) "On average how many hours do you play computer games each week?”; (2) “On average how many hours do you play computer games using a mouse each week?"; and (3) "At any stage in the past what was the maximum number of hours you played computer games in a week?" Participants were able to freely estimate the number of hours for each question.

Tests of processing speed (Gs). These tests included the following.

Digit Symbol. This test, from the Wechsler Adult Intelligence Scale, required the filling in of blank cells according to a key that was provided at the top of each test sheet. The standard 2-min time limit was used.

Visual Matching. This test, taken from the Woodcock-Johnson III Tests of Cognitive Abilities (WJ-III COG; Woodcock, McGrew, \& 


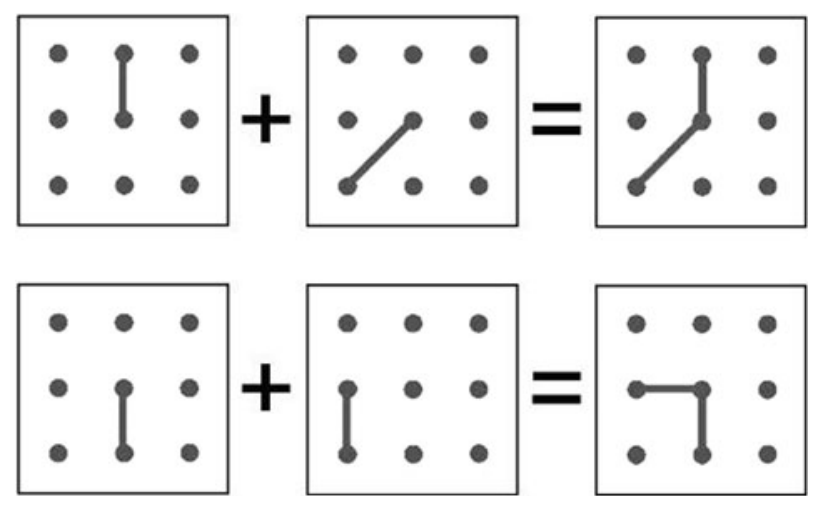

Figure 1. Matrix equation examples from dot matrix. The upper equation is the "true" example, and the lower equation is the "false" example.

Mather, 2001) required participants to search for and circle matching pairs of numbers. The standard 3-min time limit was used.

Decision Speed. This test, from the WJ-III COG, required participants to search for pairs of conceptually matched objects. The standard 3-min time limit was used.

Tests of working memory/fluid intelligence (WM/Gf). These tests included the following.

Picture Swaps. This test was adapted from the Swaps test (Stankov, 2000), which has been used as a marker test for WM in previous research (Schweizer \& Moosbrugger, 2004). This test was a computerized version, in which alphabetical symbols were replaced with colorful cartoon pictures of animals and everyday objects. Pictorial stimuli were used in order to make the test more visual. Each item consisted of a "swap" instruction screen and a response screen. Each instruction screen consisted of three pictures, presented in a row and with written instructions below asking participants to swap the order of pictures mentally. Participants could proceed to the response screen by clicking a button whenever they were ready. The response screen presented all six possible reorderings of the pictures, and participants chose the order that they thought was correct by selecting it and left-clicking the mouse. Participants had to correctly complete two practice questions with one swap and two practice sessions with two swaps prior to commencing the actual test. There were 16 items in total and three levels of difficulty; Items 1-2 required two swaps, Items 3-8 required three swaps, and Items 9-16 required four swaps. An increasing number of items was used at each successive swap level, since we expected that more difficult items would produce better differentiation in a university sample. There were no time limits imposed on any section of the test. The dependent variable was the total number of correct responses. Cronbach's alpha was .78.

Dot Matrix. This test was based on the dot matrix task (Miyake et al., 2001), which was originally based on the spatial verification task (Law, Morrin, \& Pellegrino, 1995). This task has been used as a test of visuospatial WM in a number of studies investigating the structure of WM (Colom, Abad, Rebollo, \& Shih, 2005; Colom \& Shih, 2004; Law et al., 1995). In our computerized version of the task, participants viewed a matrix equation (see Figure 1) and then verified whether they thought that the equation was true or false by left-clicking a TRUE or FALSE button. If an incorrect response was made, a pop-up warning ("No, look again closely") was displayed, and the equation was repeated. If no response was made within $8 \mathrm{sec}$, a pop-up warning ("You must verify whether the equation is true or false") was presented. This was repeated every $8 \mathrm{sec}$ if a response was not made. Following a correct response, a $5 \times 5$ grid with a dot located in one of the grid squares was presented for $1,500 \mathrm{msec}$. After a series of equation and grid displays (ranging from 2 to 5), participants were asked to indicate recalled dot locations within the
$5 \times 5$ grid. Participants responded by clicking on an empty $5 \times 5$ grid to place dots and then left-clicking the "Click to enter answer" button when they were happy with their responses. The computer program allowed participants to change their responses by clicking on a square to deselect it, and they were able to select any number of grid positions up to and including the number of grid positions presented. If a participant selected more than the appropriate number of grid positions, a pop-up warning ("Too many selected") persisted until a suitable number of grid positions had been deselected. Prior to commencing the actual test, participants had to successfully complete two practice questions, each consisting of two equation-grid pairs. The test had four levels (with 2,3,4, and 5 equation-grid pairs), and there were three items at each level. Each successive level required a larger number of grid locations to be held in memory. For example, a Level 5 item presented 5 equations, each followed by a different grid location to be remembered. There were no time limits on any section of the test, except those already mentioned. The dependent variable was the number of correctly recalled grid locations. Cronbach's alpha was .79.

Raven's Advanced Progressive Matrices Short Form (APM-SF). This was a computerized 12-item version of the original test (Raven, Raven, \& Court, 1998). The 12 items used were the subset validated by Bors and Stokes (1998) for use as a brief form. Items were presented by displaying the original stimuli on a computer screen, and the response options were numbered from 1 to 8 . Participants responded by using the computer mouse to select the number corresponding to the option they thought was correct and then clicking an "Enter" button to enter the response. The "Enter" button allowed participants to review their answer first. This was included as a measure of fluid reasoning ability (Gf). A 15-min timer was provided on the screen as a guideline, but respondents were told that they could continue if they desired. Cronbach's alpha was .70.

Computer-game-like tests. These tests included the following.

Space Code. This was the same test as the "Space Code" game described in a previous validation study (McPherson \& Burns, 2007). The screen layout for this test consisted of a spaceship cockpit, with a numerical response grid situated in the center of a control panel below a window view that looked out into space ahead (Figure 2). The grid was a $3 \times 3$ numerical grid $(6.8 \times 6.2 \mathrm{~cm})$, numbered from 1 to 9 beginning in the top left grid position. The grid sections were dark blue, and the digits were white. Each number's background turned light blue when the cursor was placed over it. To make the

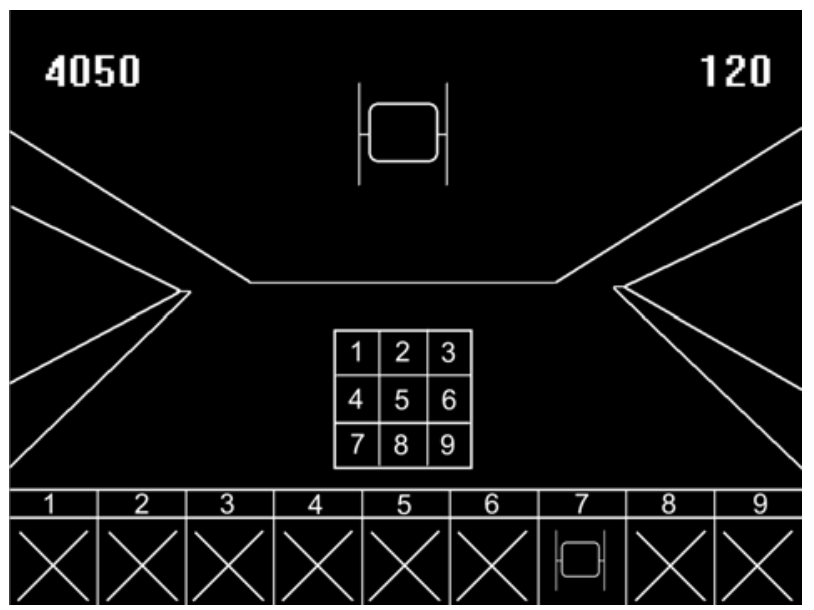

Figure 2. Basic schematic representation of screen shot from Space Code. To destroy spaceships appearing in the center of the cockpit-window view, one must fire the correct number laser on the numeric grid. The correct laser number is determined by matching the spaceship to the code in the table at the bottom of the screen. The correct laser for this item is 7 . 
grid easier to use, the cursor was restricted in such a way that it could not be moved outside of the grid area. Each item consisted of a single spaceship (approximately $5 \times 5 \mathrm{~cm}$ ) that appeared in the window view of the cockpit. At the bottom of the cockpit control panel, nine spaceships were presented, each with a single digit placed directly above it. Participants were instructed by on-screen text that correct responses (i.e., the destruction of each target spaceship) required that they click on, or "fire," the number corresponding to each spaceship at the bottom of the screen. The next item was not presented until the correct laser had been fired. Seven practice items were provided before the test began. Participants were instructed to be as fast and accurate as they could in order to destroy as many spaceships as possible in $2 \mathrm{~min}$.

Space Code also included various computer-game-like elements, which were described in an introduction screen prior to commencement of the game. The game-like elements were as follows: Spaceships were programmed to move according to a random-movement algorithm, and they zoomed in from a distant perspective point; however, no aiming was required. When the correct grid number was clicked, two laser bullets, accompanied by a "laser" sound, could be seen shooting toward the target spaceship, leading to a visible and audible explosion. When an incorrect grid number was clicked, a low-pitched descending tone was emitted, accompanied by voice-synthesized speech ("wrong laser"; Windows XP, Microsoft Sam), and the response grid was deactivated for approximately $500 \mathrm{msec}$. This was intended as an aversive consequence for rapid inaccurate firing.

A scoring system was implemented. In the beginning, participants were awarded 150 points for each correctly destroyed ship, and a cumulative total was presented in the top left portion of the screen. Up to 10 bonus stars were also awarded at predetermined score levels $(1,000,5,000$, and every multiple of 10,000 up to 80,000$)$. These were displayed above or below the score and were accompanied by a "whooshing" sound when awarded. Each star also indicated that destroyed ships would now earn a larger number of points; there was a 150-point increase for each bonus star. The current number of points that would be awarded for each ship was displayed just to the left of the mouse-response grid. In addition to providing laser and explosion sounds to accompany a correct response, Space Code increased the score in visible steps and associated these with a highpitched ascending tone. Incorrect responses were accompanied by an equivalent visible score decrement. This was further intended to make incorrect responses more aversive. A timer was situated in the top right-hand corner of the screen. The timer began at $120 \mathrm{sec}$ and counted down to indicate how much time was left, with each second decrement accompanied by a faint beep. Additionally, a verbal warning (Windows XP, Microsoft Sam) was provided at the 90-, 60-, 30-, 10-, and 5-sec marks. Background music was played during the game to further enhance the game-like feel of the test. The dependent variable was the total number of correct responses made in $2 \mathrm{~min}$. The numbers of spaceships correctly destroyed in the first and second 60 -sec blocks of the test were recorded and correlated to provide a measure of split-half reliability $(r=.75, p<.001)$.

Space Matrix. In this test, participants were asked to destroy spaceships in the same manner as in Space Code while also monitoring where dots were located on the same type of $5 \times 5$ grid as that used in Dot Matrix. These dot locations were described as indicators of which "sector" of space they were operating in, and participants were instructed that from time to time they would have to report back to headquarters which sectors they had been operating in. The screen layout was the same as that for Space Code, with the addition of the sector grid, which appeared at intervals to the right of the numerical response grid on the cockpit control panel. Sectors were presented at 7 -sec intervals, accompanied by a beep at onset, and were displayed for $2.5 \mathrm{sec}$ only. After $2-4$ sectors had been displayed, participants were asked to indicate the sectors that they recalled operating in. Participants responded by placing dots on an empty grid, as in Dot Matrix, and then clicking an "Enter Sectors" button when they were happy with their responses. Sectors could be deselected by clicking on them again. If a participant selected a smaller number of sectors than had been displayed, a pop-up warning ("Too few selected, guess if you can't remember") was presented. If a participant selected more than the appropriate number of displayed sectors, another pop-up warning ("Too many selected") was presented. Difficulty levels of the task were manipulated by increasing the number of sectors displayed before participants were asked for sector reports. Participants completed two practice runs with two sectors displayed, and one practice run with three sectors displayed. Feedback that indicated how many sectors had been correctly identified was provided.

The test consisted of 36 sector locations that were presented in sequences of 2, 3, or 4 sectors: There were six sequences of 2 sectors, four sequences of 3 sectors, and three sequences of 4 sectors. A scoring system similar to that in Space Code was implemented, but participants received a fixed 300 points for each correctly destroyed ship and 500 "bonus" points for each correctly reported sector. The bonus points were awarded after sectors had been entered, and they were displayed graphically with a scrolling score display and an accompanying sound that rose in pitch. Some elements from the original Space Code were removed to decrease the number of extraneous stimuli, since piloting suggested that the dual-task nature of the task already provided a significant amount of stimulation for players; the bonus star system was removed, and background music was replaced with a constant low humming sound akin to spaceship engine noise. The instructions emphasized that both destroying ships and reporting sectors accurately were important for the success of the mission. The primary dependent measure was the total number of correctly reported sectors out of the 36 presented throughout the test. The number of ships correctly destroyed was also obtained as a potential speed measure. The test took approximately $4.5 \mathrm{~min}$ to complete. Each sequence of sectors that was reported served as an "item," and separate scores were recorded for each sequence to provide a measure of internal consistency. Cronbach's alpha for these item units was .76.

\section{Procedure}

Participants were tested individually, or in pairs, in a quiet room and were asked to concentrate as best as they could for each individual test. We further emphasized that it was important for them to do their best on each test so that an accurate comparison could be made across their performances on all tasks. Tests were administered to all participants in the following order: nonability measures; Space Code; Space Matrix; Visual Matching; Decision Speed; Digit Symbol; Dot Matrix; Picture Swaps; APM-SF; Space Code; Space Matrix. A consistent test order was used in an effort to make any fatigue or motivational effects comparable across all participants and to provide the largest possible gap between the first and second administrations of Space Code and Space Matrix, which were included to provide some estimate of task stability. Sessions took approximately $2 \mathrm{~h}$ to complete.

All computerized tests were run on a Pentium 4 computer $(2.4 \mathrm{MHz})$ with a Microsoft optical Intellimouse. They were displayed on a 19-in. CRT monitor with a vertical refresh rate of $100 \mathrm{~Hz}$ and a screen resolution of $1,280 \times 1,024$ pixels.

\section{Results}

\section{Descriptive Statistics}

Descriptive statistics for all tests are presented in Table 1. Analysis of the means for the two administrations of Space Code revealed a substantial practice effect $[t(65)=23.74, p<.001, d=2.29]$. The effect size for the mean difference was approximately twice the size that it was in a previous study (McPherson \& Burns, 2007), a difference that was probably due to the participants' increased exposure to the codes gained in Space Matrix. The correlation between the two sets of test scores $(r=$ $.75, p<.001)$ suggested that Space Code was reliable across the two administrations. There was a smaller, but 
Table 1

Descriptive Statistics for Ability and Computer-Game-Like Tests (Experiment 1)

\begin{tabular}{|c|c|c|c|c|}
\hline & $M$ & $S D$ & Min. & Max. \\
\hline Digit Symbol & 87.4 & 13.3 & 50 & 115 \\
\hline Visual Matching a & 559.3 & 26.0 & 510 & 625 \\
\hline Decision Speed ${ }^{a}$ & 541.2 & 19.5 & 499 & 586 \\
\hline Picture Swaps & 11.9 & 3.1 & 6 & 16 \\
\hline Dot Matrix & 32.2 & 5.7 & 20 & 42 \\
\hline $\mathrm{APM}-\mathrm{SF}$ & 6.4 & 2.7 & 1 & 12 \\
\hline Space Code & 61.0 & 9.1 & 40 & 90 \\
\hline Space Codeb & 87.5 & 13.6 & 58 & 111 \\
\hline Space Matrix & 19.3 & 6.2 & 8 & 34 \\
\hline Space Matrix ${ }^{b}$ & 22.8 & 7.2 & 9 & 36 \\
\hline Space Matrix speed & 111.8 & 31.0 & 57 & 187 \\
\hline Space Matrix speed ${ }^{b}$ & 134.9 & 26.4 & 80 & 182 \\
\hline
\end{tabular}

Note-APM-SF is Raven's Advanced Progressive Matrices Short Form. Space Matrix speed is the number of ships destroyed in Space Matrix. $\quad$ aBased on Rasch scaled $W$ scores centered on 500. bScores from second administration of test.

significant, practice effect for Space Matrix $[t(65)=5.26$, $p<.001, d=0.52]$, and the correlation between the two administrations was also quite strong for Space Matrix $(r=.77, p<.001)$. The difficulty level for Space Matrix appeared to be well calibrated for this sample; the mean score for both administrations was slightly larger than half of the maximum possible score of 36 . There was also a significant practice effect for Space Matrix speed, the speed measure taken from Space Matrix $[t(66)=15.10, p<$ $.001, d=0.80]$, and there was a very strong relationship between the two administrations $(r=.93, p<.001)$.

\section{Test Intercorrelations}

Correlations between all ability tests are presented in Table 2. The relationships between the Gs and WM/Gf marker tests were generally as we expected. The three Gs marker tests-Digit Symbol, Visual Matching, and Decision Speed-were all significantly correlated with each other, as were the three WM/Gf tests-Picture Swaps, Dot Matrix, and APM-SF. Correlations between the two types of marker tests were generally smaller, but most correlations were statistically significant. Both computer-gamelike tests had substantial correlations with a number of traditional tests, and these were of a magnitude similar to that of the correlations between the marker tests themselves. Space Matrix speed was strongly related to Space Code but not to the primary Space Matrix memory measure, thus indicating that the speed and WM components of the test were independent.

\section{Exploratory Factor Analyses (EFA)}

The tests included in EFA procedures were the three Gs markers (Digit Symbol, Visual Matching, and Decision Speed), the three WM/Gf measures (Picture Swaps, Dot Matrix, and APM-SF), and the two computer-gamelike tests (Space Code and Space Matrix). The APM-SF was expected to load with the WM tasks in the absence of other Gf marker tests. The Kaiser-Meyer-Olkin (.841) and Bartlett $\left[\chi^{2}(28)=216.85, p<.001\right]$ statistics indicated that the correlation matrix was suitable for EFA. An initial principal components analysis resulted in two components with eigenvalues larger than unity (4.02 and 1.32 , respectively) and a third eigenvalue of 0.64 . The first two components accounted for $50.2 \%$ and $16.5 \%$ of the total variance, respectively. To assess latent construct validity specifically, however, subsequent analyses were conducted using principal axis factoring (PAF).

The number of factors to extract was assessed using multiple decision criteria (Fabrigar, Wegener, MacCallum, \& Strahan, 1999; Henson \& Roberts, 2006). These were the scree test (Cattell, 1966), parallel roots analysis (PRA; Montanelli \& Humphreys, 1976), and Velicer's (1976) minimum average partial (MAP), as well as a consideration of the residual correlations and the theoretical issues relevant to the present study. PRA was conducted for 1,000 random permutations of the raw data, using SPSS syntax by O'Connor (2000), and the MAP test was performed using SPSS syntax for extension analyses, also by O'Connor (2001).

Visual inspection of the scree plot and both the PRA and MAP criteria indicated two factors. A two-factor PAF solution was obtained, explaining $56.16 \%$ of the common variance. The two factors were then rotated using the oblique direct oblimin procedure $(\Delta=0)$. The correlation between the two rotated factors was .50 . There were no residual correlations larger than .10, and the largest residual correlation was between Dot Matrix and Space Matrix $(r=.09)$. Inspection of the pattern matrix (Table 3$)$ indicated two quite distinct factors. Factor 1 was strongly loaded by all of the WM/Gf marker tests and Space Matrix. Factor 2 was strongly loaded by all of the paper-and-

Table 2

Correlation Matrix for Ability and Computer-Game-Like Tests (Experiment 1)

\begin{tabular}{|c|c|c|c|c|c|c|c|c|c|}
\hline Test & 1 & 2 & 3 & 4 & 5 & 6 & 7 & 8 & 9 \\
\hline 1. Digit Symbol & - & & & & & & & & \\
\hline 2. Visual Matching & $.57^{* *}$ & - & & & & & & & \\
\hline 3. Decision Speed & $.53^{* *}$ & $.59^{* *}$ & - & & & & & & \\
\hline 4. Picture Swaps & $.36^{* *}$ & $.34^{* *}$ & .23 & - & & & & & \\
\hline 5. Dot Matrix & $.39^{* *}$ & $.46^{* *}$ & $.43^{* *}$ & $.54^{* *}$ & - & & & & \\
\hline 6. APM-SF & $.30^{*}$ & $.24^{*}$ & .11 & $.63^{* *}$ & $.48^{* *}$ & - & & & \\
\hline 7. Space Codea & $.45^{* *}$ & $.48^{* *}$ & $.37^{* *}$ & $.37^{* *}$ & $.41^{* *}$ & $.42^{* *}$ & - & & \\
\hline 8. Space Matrix ${ }^{a}$ & $.33^{* *}$ & $.42^{* *}$ & $.26^{*}$ & $.54^{* *}$ & $.66^{* *}$ & $.53^{* *}$ & $.51^{* *}$ & - & \\
\hline 9. Space Matrix speed ${ }^{a}$ & $.29^{*}$ & $.27^{*}$ & $.34^{* *}$ & .21 & .15 & $.25^{*}$ & $.79^{*}$ & .15 & - \\
\hline
\end{tabular}

Note-APM-SF is Raven's Advanced Progressive Matrices Short Form. a Scores are for first administration of test. ${ }^{*} p<.05 .{ }^{* *} p<.01$. 
Table 3

Summary of Two-Factor PAF Solution (Experiment 1)

\begin{tabular}{|c|c|c|c|c|c|}
\hline & \multicolumn{2}{|c|}{ Pattern Matrix } & \multicolumn{2}{|c|}{ Structure Matrix } & \multirow[b]{2}{*}{$h^{2}$} \\
\hline & Factor 1 & Factor 2 & Factor 1 & Factor 2 & \\
\hline Digit Symbol & .121 & .636 & .436 & .696 & .495 \\
\hline Visual Matching & .068 & .769 & .449 & .803 & .648 \\
\hline Decision Speed & -.121 & .828 & .289 & .769 & 671 \\
\hline Picture Swaps & .755 & .002 & .756 & .376 & .572 \\
\hline Dot Matrix & .580 & .268 & .712 & .555 & .562 \\
\hline APM-SF & .837 & -.148 & .764 & .267 & .679 \\
\hline Space Code & .375 & .368 & .558 & .554 & .413 \\
\hline Space Matrix & .716 & .108 & .769 & .462 & .601 \\
\hline Eigenvalue $^{\mathrm{a}}$ & 3.04 & 2.76 & 3.04 & 2.76 & \\
\hline$\%$ of variance ${ }^{b}$ & 38.01 & 34.52 & 38.01 & 34.52 & \\
\hline (Cumulative \%) & & $(56.10)$ & & $(56.10)$ & \\
\hline
\end{tabular}

Note-Bold type indicates factor loadings $>.4$. APM-SF is Raven's Advanced Progressive Matrices Short Form. aEigenvalues presented are for extracted factors after rotation. ${ }^{\mathrm{b}}$ Indicates percentage of total variance accounted for by rotated factors.

pencil Gs tests. Space Code was not strongly loaded on either factor. Because the two factors were quite strongly correlated, however, it is important to consider the structure matrix also (Henson \& Roberts, 2006). As can be seen in Table 3, Space Code was similarly correlated with both factors. Digit Symbol and Visual Matching were very strongly correlated with Factor 2, but also had substantial correlations with Factor 1. Similarly, Dot Matrix and Space Matrix were strongly correlated with Factor 1 but also had substantial correlations with Factor 2. Despite an apparent degree of interrelatedness between the factors, it seems reasonable to interpret them as two hypothesized constructs; Factor 1 can thus be interperted as WM/Gf and Factor 2 can be interpreted as Gs. Considering Space Code's shared loadings, however, it was decided that a Schmid-Leiman transformation (Schmid \& Leiman, 1957) would provide further aid in the interpretation of these results.

The Schmid-Leiman transformation procedure enables one to assess the independent contributions of first- and second-order factors on the variables (Carroll, 1993; Schmid \& Leiman, 1957). The two-factor EFA solution was subjected to a Schmid-Leiman transformation, using syntax developed by Wolff and Preising (2005). The obtained solution is presented in Table 4. The higher order general factor explained more than one quarter of the total variance, and all of the variables had substantial loadings on this factor. Additionally, the first-order factors were more clearly defined after the general factor loadings had been taken into account. A first-order WM/Gf factor was clearly loaded by Picture Swaps, Dot Matrix, and APMSF, and a first-order Gs factor was clearly loaded by the paper-and-pencil speed tests. Space Code was substantially loaded on the general factor but had relatively small and almost equal loadings on both first-order factors.

\section{Composite Ability Score Analyses}

To assess the relationship between the computer-gamelike tests and the traditional ability tests more directly, composite Gs and WM/Gf scores were derived and were correlated with the computer-game-like tests. Gs com- posites were derived by computing the average $z$ scores of Digit Symbol, Visual Matching, and Decision Speed scores. A WM/Gf composite was formed using the Picture Swaps, Dot Matrix, and APM-SF $z$ scores. Space Code was somewhat similarly correlated with both the Gs $(r=.52, p<.001)$ and WM/Gf $(r=.47, p<.001)$ composites. Space Matrix was very strongly correlated with the WM/Gf composite $(r=.69, p<.001)$ but more moderately correlated with the Gs composite $(r=.40$, $p<.001$ ). The Gs and $\mathrm{WM} / \mathrm{Gf}$ composite measures were correlated to the same extent as were their respective EFA factors $(r=.45, p<.01)$.

\section{Correlational Analyses of Nonability and Ability Measures}

The relationships of demographic and gaming measures with ability composite scores and the game-like computerized tests were examined using correlations. Inspection of the correlations between gaming measures indicated that Items 1 ("On average how many hours do you play computer games each week?") and 2 ("On average how many hours do you play computer games using a mouse each week?") were very strongly correlated $(r=.92, p<.001)$, whereas Item 3 ("At any stage in the past what was the maximum number of hours you played computer games in a week?") was more moderately correlated with Items 1 and $2(r=.45, p<.001$, and $r=.26, p<.05$, respectively). Items 1 and 2 were combined to create a game play measure, and Item 3 was retained as a separate measure, past game play. Gender was significantly correlated with game play $(r=.47, p<.001)$ and past game play $(r=.35$, $p<.001)$, the positive correlation indicating that males had more game experience. Game experience measures were not significantly correlated with either age or handedness. Correlations between these measures and ability measures are presented in Table 5. Gender was moderately correlated with both WM/Gf and Space Code. Game play was moderately correlated with all ability measures except Gs, and past game play was moderately correlated with WM/Gf and somewhat more strongly correlated with Space Code. To assess whether the relationship between gender and the computerized speed tests was mediated by gaming experience, partial correlations were obtained.

Table 4

Summary of Schmid-Leiman Hierarchical Solution (Experiment 1)

\begin{tabular}{llrrr}
\hline & \multicolumn{1}{l}{ G } & WM/Gf & \multicolumn{1}{l}{ Gs } & $h^{2}$ Total \\
\hline Digit Symbol & $\mathbf{. 5 3 2}$ & .086 & $\mathbf{. 4 5 2}$ & .495 \\
Visual Matching & $\mathbf{. 5 8 8}$ & .048 & $\mathbf{. 5 4 7}$ & .648 \\
Decision Speed & $\mathbf{. 4 9 7}$ & -.086 & $\mathbf{. 5 8 9}$ & .601 \\
Picture Swaps & $\mathbf{. 5 3 2}$ & $\mathbf{. 5 3 7}$ & .001 & .572 \\
Dot Matrix & $\mathbf{. 5 9 6}$ & $\mathbf{. 4 1 2}$ & .191 & .562 \\
APM-SF & $\mathbf{. 4 8 4}$ & $\mathbf{. 5 9 5}$ & -.105 & .600 \\
Space Code & $\mathbf{. 5 2 2}$ & .267 & .262 & .412 \\
Space Matrix & $\mathbf{. 5 7 9}$ & $\mathbf{. 5 0 9}$ & .077 & .601 \\
Eigenvalue & 2.36 & 1.16 & 0.97 & 4.49 \\
\% of variance & 29.5 & 14.5 & 12.1 & 56.1 \\
\hline
\end{tabular}

Note-Bold type indicates factor loadings >.4. APM-SF is Raven's Advanced Progressive Matrices Short Form. aPercentage of total variance accounted for. 
Table 5

Correlations of Nonability Measure With Ability Composite Scores and Computer-Game-Like Tests (Experiment 1)

\begin{tabular}{lrccc}
\hline & Gs & WM/Gf & $\begin{array}{c}\text { Space } \\
\text { Code }\end{array}$ & $\begin{array}{c}\text { Space } \\
\text { Matrix }\end{array}$ \\
\hline Age & .00 & -.04 & -.05 & .02 \\
Gender & -.15 & $.26^{*}$ & $.25^{*}$ & .20 \\
Handedness & .08 & .03 & -.12 & -.12 \\
Game play & .05 & $.30^{*}$ & $.31^{*}$ & $.29^{*}$ \\
Past game play & -.04 & $.25^{*}$ & $.42^{* *}$ & .10 \\
\hline
\end{tabular}

aGender was coded in such a way that positive correlations indicated higher scores for males and that negative correlations indicated higher scores for females. ${ }^{b}$ Handedness was coded in such a way that positive correlations indicated higher scores for right-handed participants. ${ }^{*} p<$ $.05 .{ }^{* *} p<.01$.

After game play and past game play were controlled for, the correlation between gender and Space Code was nonsignificant and almost zero $(r=.06, p=.65)$.

\section{EXPERIMENT 2}

In Experiment 1 and previous studies (McPherson \& Burns, 2005, 2007), we assessed the viability of using computer-game-like tests of cognitive abilities within university samples. We have also suggested that computergame-like tests may be particularly suitable for testing younger children. Indeed, the initial impetus for our research program came from our own frequent observations of boredom and motivational fatigue in school-aged children undertaking more traditional assessments via computer. In Experiment 2, we thus aimed to assess the concurrent validity of Space Code and Space Matrix within a school-aged sample. Additionally, we also sought to make a preliminary assessment of the external validity of the two computer-game-like tests.

In their recent large-scale study, Luo et al. (2006) demonstrated that Gs and WM measures predicted academic achievement as well as traditional Gc (crystalized ability) and Gf measures did. Perhaps this is not altogether surprising considering the substantial amount of research that indicates that Gs and WM measures share substantial variance with broader composite cognitive ability and/or Gf itself (see McGrew, 2005, for a review). It is important to note, however, that a substantial portion of the relationship between Gs and more general ability is often indirect, being mediated by relationships with both short-term memory (Gsm) and WM. Additionally, it is possible that Gs and WM measures will differ in their relationships to specific areas of achievement (Floyd, Shaver, \& McGrew, 2003).

In addition to assessing concurrent validity, therefore, we aimed to assess the external validity of Space Code and Space Matrix as predictors of scholastic achievement. In order to address the possibility that Gs and WM measures may be differentially related to various achievement domains, furthermore, we used three external criterion measures: English, mathematics, and science subject grades. Although specific predictions were difficult to make, we did have some expectations that were based on results from the Woodcock-Johnson III normative sample reported by
Floyd, Shaver, and McGrew (2003). We expected Gs measures to predict English grades better than they would predict mathematics or science grades. We also anticipated that WM measures, to the extent that they share predictive power with Gf measures, would predict mathematics grades better than they would predict English or science grades. Furthermore, the stronger direct relationship between WM and more general ability suggested that WM measures may be stronger predictors than Gs measures in general. Our primary aim, however, was to directly compare the external validity of the computer-game-like tests and that of established ability-marker tests.

\section{Method}

\section{Participants}

There were 94 participants, all children 11 to 14 years of age (mean $=12.88, S D=0.80)$ who were recruited from 6th, 7th, and 8 th grades at a private school in Adelaide, South Australia. There were 42 males (mean $=12.73$ years, $S D=0.87)$ and 52 females (mean $=13.00$ years, $S D=0.73$ ).

\section{Materials}

Nonability measures. The measures were the same as those described in Experiment 1, except for age, which was measured in years and months.

Tests of processing speed (Gs). These measures included the following.

Digit Symbol. As described in Experiment 1.

Visual Matching. As described in Experiment 1.

Decision Speed. As described in Experiment 1.

Tests of working memory and fluid intelligence (WM/Gf). These measures included the following.

Picture Swaps. This test was the same as that described in Experiment 1 , with alterations made to accommodate the younger sample and stricter time limits. There were 24 items in total and four levels of difficulty; Items 1-6 required one swap, Items 7-12 required two swaps, Items 13-18 required three swaps, and Items 19-24 required four swaps. There was a 10 -min time limit, implemented within the computer software. The dependent variable was the total number of correct responses completed within the time limit. Cronbach's alpha was .87 .

Raven's Standard Progressive Matrices Short Form (SPM-SF). This was a computerized, 32-item version of Raven et al.'s (1998) original test. The 32 items used were the same subset used by Zajac and Burns (2007), with items selected on the basis of prior research that investigated the factor structure of this test (Lynn, Allik, \& Irwing, 2004). Items were presented and were responded to in the same manner as was used in the APM-SF described in Experiment 1. There was a 10-min time limit, implemented within the computer software. The dependent variable was the total number of correct responses completed within the time limit. Cronbach's alpha was 69 .

Computer-game-like tests. These measures included the following.

Space Code. This was the same test as that described in Experiment 1 . As in Experiment 1, the numbers of spaceships correctly destroyed in the first and second 60 -sec blocks of the test were recorded and were correlated to provide a measure of split-half reliability $(r=.77, p<.001)$.

Space Matrix. This was the same test as that described in Experiment 1 , with the following alterations made to accommodate the younger sample. There were two practice runs with 1 sector displayed, and one practice run with 2 sectors displayed. The test consisted of 30 sector locations that were presented in sequences of 2 or 3 sectors: There were six sequences of 2 sectors and six sequences of 3 sectors. The test took approximately $4 \mathrm{~min}$ to complete. Cronbach's alpha was .70.

Academic achievement. These measures included the following. 
Table 6

Descriptive Statistics for Ability and Computer-Game-Like Tests (Experiment 2)

\begin{tabular}{lrrrr}
\hline & $M$ & \multicolumn{1}{c}{$S D$} & Min. & Max. \\
\hline Digit Symbol & 60.3 & 12.1 & 31 & 110 \\
Visual Matching $^{\text {a }}$ & 520.2 & 20.8 & 473 & 564 \\
Decision Speed $^{\text {a }}$ & 517.0 & 15.3 & 480 & 543 \\
Picture Swaps $_{\text {SPM-SF }}$ & 11.45 & 5.0 & 1 & 24 \\
Space Code & 16.3 & 4.0 & 8 & 25 \\
Space Matrix & 49.1 & 8.7 & 20 & 73 \\
Space Matrix speed & 13.1 & 4.6 & 4 & 27 \\
\hline
\end{tabular}

Note-SPM-SF is Raven's Standard Progressive Matrices Short Form. Space Matrix speed is the number of ships destroyed in Space Matrix. $\quad$ aBased on Rasch scaled $W$ scores centered on 500 .

Preparatory school grades. For students in 6th and 7th grades $(n=44)$, first-semester school subject scores were obtained for English, mathematics, and science. These grades were in the form of ordinal categorical grades (e.g., A, B, or C). These grades were recoded as the minimum percentages required for each grade $(\mathrm{A}=85$, $\mathrm{B}=70, \mathrm{C}=55)$. No student had obtained a grade lower than a $\mathrm{C}$.

High-school grades. For students in 8th grade $(n=50)$, firstsemester school subject scores were obtained for English, mathematics, and science. These grades were raw percentages.

\section{Procedure}

Teachers distributed information sheets and parental or guardian consent forms to children within class. Students were asked to read the information sheet with their parent or guardian and to return the signed consent form if they were interested in participating. The information sheet stressed that participation was voluntary, that it was not associated with school assessment in any way, and that results would be anonymous and confidential.

Participants were tested in groups of 2 to 4 in a quiet room and were asked to concentrate and do their best for each individual test. Tests were administered to all participants in the following order: nonability measures; Space Code; Space Matrix; Visual Matching; Decision Speed; Digit Symbol; Picture Swaps; and SPM-SF. A consistent test order was used in an effort to make any fatigue or motivational effects consistent across all participants. The sessions took approximately $45 \mathrm{~min}$ to complete.

All computerized tests were run on a Pentium 4 computer $(2.4 \mathrm{MHz})$ with a Microsoft optical Intellimouse. They were displayed on a 19 -in. LCD monitor with a vertical refresh rate of $100 \mathrm{~Hz}$ and a screen resolution of $1,280 \times 1,024$ pixels.

\section{Results}

Descriptive statistics for all tests are presented in Table 6. Both Picture Swaps and SPM-SF had mean scores equal to about half the possible maximum scores
(24 and 32, respectively). The mean score for Space Matrix was also slightly below half of the possible maximum score (30). An independent-samples $t$ test indicated that the Space Code mean for the Experiment 1 sample $(M=61.0, S D=9.1)$ was higher than that for the present sample $[t(162)=8.50, p<.001, d=1.34]$. The Experiment 1 sample also had higher mean scores for Digit Symbol $[t(162)=13.60, p<.001, d=2.15]$, Visual Matching $[t(162)=10.69, p<.001, d=1.69]$, and Decision Speed $[t(162)=8.90, p<.001, d=1.41]$. These results are consistent with research that has indicated clear developmental increases in Gs across these age groups (Horn \& Noll, 1997). The smaller effect size for Space Code suggests that the task was not particularly difficult, relative to the other Gs tasks, for the younger sample. Comparisons for other tests could not be made, because these were not equivalent for both samples.

\section{Test Intercorrelations}

Correlations between all ability measures are presented in Table 7. The relationships between the Gs and WM/Gf marker tests were generally as we expected, although the magnitude of correlations was generally smaller than in Experiment 1. The three Gs marker tests-Digit Symbol, Visual Matching, and Decision Speed-were all significantly correlated with each other, as were the two WM/ Gf tests, Picture Swaps and SPM-SF. Picture Swaps also showed substantial relationships with two Gs marker tests, Digit Symbol and Visual Matching. Space Code was significantly correlated with only Digit Symbol and SPMSF, whereas Space Matrix was significantly correlated with every other test. Correlations between Space Matrix and ability tests were of a magnitude similar to the correlations between the marker tests themselves, whereas these correlations were smaller for Space Code. As in Experiment 1, Space Matrix speed was strongly related to Space Code but not to the primary Space Matrix memory measure, once again indicating that the speed and WM components of the test were independent.

\section{Exploratory Factor Analyses}

The tests included in EFA procedures were the three Gs markers (Digit Symbol, Visual Matching, and Decision Speed), the two WM/Gf measures (Picture Swaps and SPM-SF), and the two computer-game-like tests (Space Code and Space Matrix). As with the APM-SF

Table 7

Correlation Matrix for Ability and Computer-Game-Like Tests (Experiment 2)

\begin{tabular}{|c|c|c|c|c|c|c|c|c|}
\hline Test & 1 & 2 & 3 & 4 & 5 & 6 & 7 & 8 \\
\hline 1. Digit Symbol & - & & & & & & & \\
\hline 2. Visual Matching & $.57^{* *}$ & - & & & & & & \\
\hline 3. Decision Speed & $.42^{* *}$ & $.40^{* *}$ & - & & & & & \\
\hline 4. Picture Swaps & $.32^{* *}$ & $.33^{* *}$ & .14 & - & & & & \\
\hline 5. SPM-SF & $.23^{*}$ & .15 & .19 & $.38^{* *}$ & - & & & \\
\hline 6. Space Code & $.29^{* *}$ & .16 & .20 & .20 & $.36^{* *}$ & - & & \\
\hline 7. Space Matrix & $.31^{* *}$ & $.23^{*}$ & $.22 *$ & $.35^{* *}$ & $.44^{* *}$ & $.34^{* *}$ & - & \\
\hline 8. Space Matrix speed & .03 & -.03 & -.11 & .15 & $.24^{*}$ & $.61^{* *}$ & .05 & - \\
\hline
\end{tabular}


in Experiment 1, the SPM-SF was expected to load with the WM tasks in the absence of other Gf marker tests. The Kaiser-Meyer-Olkin (.764) and Bartlett $\left[\chi^{2}(21)=\right.$ $127.59, p<.001]$ statistics indicated that the correlation matrix was suitable for EFA. An initial principal components analysis resulted in two components with eigenvalues larger than unity (2.80 and 1.21 , respectively) and a third eigenvalue of 0.85 . The first two components accounted for $40.0 \%$ and $17.4 \%$ of the total variance, respectively. As in Experiment 1, subsequent analyses were conducted using PAF.

The number of factors to extract was decided using the same criteria as those used in Experiment 1. Visual inspection of the scree plot and parallel roots analysis suggested a two-factor solution, whereas Velicer's MAP test suggested a one-factor solution. A one-factor PAF solution was judged unsuitable because there were 10 residual correlations (47.6\%) larger than .10. A two-factor PAF solution was next obtained, explaining $43.1 \%$ of the common variance. The two factors were then rotated using the oblique direct oblimin procedure $(\Delta=0)$. The correlation between the two factors was -.50 . There were no residual correlations larger than .10. Inspection of the pattern matrix (Table 8) indicates two quite distinct factors. Factor 1 was strongly loaded by SPM-SF, Space Matrix, and, to a lesser degree, Picture Swaps and Space Code. Factor 2 was strongly loaded by Digit Symbol and Visual Matching, and Decision Speed had a smaller, but substantial, loading. Considering the structure matrix, it can be seen that all of the tests were at least moderately correlated with both factors, but the pattern of relationships was similar to that in the pattern matrix. Factor 1 was most strongly loaded by SPM-SF, Space Matrix, and Picture Swaps and was therefore interpreted as a WM/ Gf factor. Factor 2 was most strongly loaded by Visual Matching, Digit Symbol, and Decision Speed and was therefore interpreted as a Gs factor. These results further support the concurrent validity of Space Matrix as a measure of WM/Gf. Because Space Code loaded more strongly on the WM/Gf in both the pattern and structure matrix, however, the concurrent validity of Space Code as a Gs measure was not supported.

Table 8

Summary of Two-Factor PAF Solution (Experiment 2)

\begin{tabular}{|c|c|c|c|c|c|}
\hline & \multicolumn{2}{|c|}{ Pattern Matrix } & \multicolumn{2}{|c|}{ Structure Matrix } & \multirow[b]{2}{*}{$h^{2}$} \\
\hline & Factor 1 & Factor 2 & Factor 1 & Factor 2 & \\
\hline Digit Symbol & .081 & -.729 & .445 & -.769 & .597 \\
\hline Visual Matching & -.081 & -.813 & .325 & -.772 & .654 \\
\hline Decision Speed & .061 & -.487 & .304 & -.518 & .271 \\
\hline Picture Swaps & .418 & -.178 & .507 & -.387 & .281 \\
\hline SPM-SF & .811 & .151 & .735 & -.254 & .634 \\
\hline Space Code & .463 & -.064 & .496 & -.296 & .249 \\
\hline Space Matrix & .615 & -.056 & .643 & -.363 & .416 \\
\hline Eigenvalue $^{\mathrm{a}}$ & 1.85 & 1.89 & 1.85 & 1.89 & \\
\hline$\%$ of variance b & 26.47 & $\begin{array}{c}26.99 \\
(41.30)\end{array}$ & 26.47 & $\begin{array}{c}26.99 \\
(4130)\end{array}$ & \\
\hline
\end{tabular}

Note-Bold type indicates factor loadings $>.4$. SPM-SF is Raven's

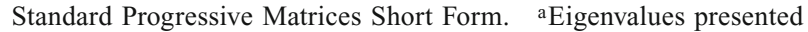
are for extracted factors after rotation. bIndicates percentage of total variance accounted for by rotated factors.

\section{Composite Ability Score Analyses}

To assess the relationship between the computer-gamelike tests and the traditional ability tests more directly, composite Gs and WM/Gf scores were derived and were correlated with the computer-game-like tests. Gs composites were derived by computing average $z$ scores for Digit Symbol, Visual Matching, and Decision Speed scores. A WM/Gf composite was formed using the Picture Swaps and SPM-SF $z$ scores. Space Code was more strongly correlated with the WM/Gf composites $(r=.34, p<.001)$ than it was with the Gs composite $(r=.27, p<.01)$. Space Matrix was quite strongly correlated with the WM/ Gf composite $(r=.48, p<.001)$ and moderately correlated with the Gs composite $(r=.31, p<.001)$. The Gs and WM/Gf composite measures were less strongly correlated than were the respective EFA factors $(r=.34$, $p<.01)$.

\section{Correlational Analyses of Nonability and Ability Measures}

The relationships of demographic and gaming measures with ability composite scores and the game-like computerized tests were examined using correlations. Inspection of the correlations between gaming measures indicated that Items 1 ("On average how many hours do you play computer games each week?") and 2 (“On average how many hours do you play computer games using a mouse each week?") were strongly correlated ( $r=.73$, $p<.001$ ), whereas Item 3 ("At any stage in the past what was the maximum number of hours you played computer games in a week?") was strongly correlated with Item 1 $(r=.69, p<.001)$ but more moderately correlated with Item $2(r=.42, p<.001)$. To make results consistent with Experiment 1, Items 1 and 2 were combined to create a game play measure, and Item 3 was retained as a separate measure, past game play. Gender was significantly correlated with game play $(r=.38, p<.001)$ and past game play $(r=.25, p<.05)$, and the positive correlation indicated that males had more game experience. Game experience measures were not significantly correlated with either age or handedness. Correlations between the nonability measures and ability measures are presented in Table 9. In keeping with well-documented developmental trends within these age groups (Horn \& Noll, 1997), age was significantly correlated with Gs, WM/Gf, and Space Code. There was no significant relationship with Space Matrix, however. Gender was not significantly correlated with any of the ability measures. Game play was correlated with WM/Gf and Space Code.

\section{External Validity Comparisons}

To assess the external predictive validity of the computer-game-like tests, correlations were obtained between these tasks and school grades (see Table 10). Additionally, traditional marker tests for Gs and WM/ Gf were chosen to provide comparative predictors. Digit Symbol was chosen as a comparative task for Space Code because it is a well-known Gs task from what is perhaps the most widely known and used IQ battery, the Wechsler scales, and because both Space Code and Digit Symbol 
Table 9

Correlations of Nonability Measures With Ability Composite Scores and Computer-Game-Like Tests (Experiment 2)

\begin{tabular}{lcccc}
\hline & Gs & WM/Gf & $\begin{array}{c}\text { Space } \\
\text { Code }\end{array}$ & $\begin{array}{c}\text { Space } \\
\text { Matrix }\end{array}$ \\
\hline Age & $.33^{* *}$ & $.40^{* *}$ & $.26^{*}$ & .14 \\
Gender $^{\mathrm{a}}$ & -.18 & .06 & .18 & .14 \\
Handedness $^{\mathrm{b}}$ & .14 & .01 & .07 & -.10 \\
Game play $_{\text {Past game play }}$ & .04 & $.22^{*}$ & $.25^{*}$ & .14 \\
\hline
\end{tabular}

aGender was coded in such a way that positive correlations indicated higher scores for males and negative correlations indicated higher scores for females. ${ }^{b}$ Handedness was coded in such a way that positive correlations indicated higher scores for right-handed participants. ${ }^{*} p<$ .05. ${ }^{* *} p<.01$.

are coding tasks. The SPM-SF was chosen as a comparative task for Space Matrix because it is perhaps the most widely recognized test of Gf and is thought to be one of the best indicators of general cognitive ability (Carpenter, Just, \& Shell, 1990; Fry \& Hale, 1996; Jensen, 1998; Prokosch, Yeo, \& Miller, 2005). Kendall's tau correlations were used to assess the relationships with preparatory subject grades because these were categorical and there were numerous tied ranks (Howell, 2007).

Space Code showed no significant relationships with school subject grades in either sample. Digit Symbol had a moderate significant relationship with preparatory English grades. Considering SPM-SF and Space Matrix, it can be seen that the WM tasks were generally better predictors of school grades than were the speed tasks. SPM-SF and Space Matrix were significant predictors of preparatory English grades but not of high-school English grades. The only other significant correlation was between Space Matrix and mathematics grades in both samples. Correlations between SPM-SF and mathematics grades were nonsignificant but only slightly smaller in magnitude. Space Matrix had the strongest correlations with all subject grades except for high-school English and science.

\section{GENERAL DISCUSSION}

The results of both experiments provide preliminary support for the idea of developing computer-game-like tests of information-processing abilities. In both samples, Space Code and Space Matrix were found to have acceptable internal reliabilities, and, within the university sample, both tests provided stable results across two ad- ministrations. The computer-game-like tests also shared substantial variance with the traditional abilities tests; however, there were varying levels of support for each test's concurrent and external validity.

In Experiment 1, Space Code loaded, relatively weakly, on both a Gs and a WM/Gf factor in the initial EFA solution. In a subsequent Schmid-Leiman transformation, Space Code had a substantial loading on the higher order factor but relatively weaker loadings on the first-order Gs and WM/Gf factors. Thus, although Space Code appeared to share considerable variance with the traditional tests, it did not exhibit discriminant validity as a measure of Gs. In Experiment 2, Space Code actually loaded more strongly on a WM/Gf factor. In an earlier article (McPherson \& Burns, 2007), we reported that a simple prototype version of Space Code loaded on a Gs and a visuospatial ability (Gv) factor. In a second study incorporating a larger battery of tests, however, the final version of Space Code was primarily loaded on a Gs factor. Overall, these results suggest Space Code is probably a mixed-ability measure and will require further refinement if it is to function as a measure of Gs.

Space Code was originally based on a speeded, computerized coding task, Symbol Digit, that utilized simple symbols similar to those used in the well-known Digit Symbol subtest from the various Wechsler scales (McPherson \& Burns, 2005). The Symbol Digit test was found to load saliently on a Gs factor across three separate studies. Space Code and Symbol Digit use the same presentation and response methods, but Space Code uses multicolored spaceships of various designs as coding symbols. For this reason, we suggest that the more complex loading patterns observed for Space Code are probably due to the use of more visually complex stimuli. As pointed out by a reviewer of the present article, the use of more complex visual stimuli would also make greater demands on visual memory, which may also explain why the task had considerable loadings on the WM/Gf factor. We believe that the mixed loadings are less likely to be due to the inclusion of dynamic game-like elements, such as scoring and auditory feedback, because loadings on a Gv factor were observed for a prototype version that did not include these elements (McPherson \& Burns, 2007).

In contrast to Space Code, Space Matrix exhibited quite consistent psychometric properties across both experiments. In Experiments 1 and 2, Space Matrix exhibited a pattern of loadings similar to that for the traditional WM

Table 10

Correlations for Comparative Ability and Computer-Game-Like Tests With School Subject Grades (Experiment 2)

\begin{tabular}{lccccccc}
\hline & \multicolumn{2}{c}{ Preparatory School Grades } & & \multicolumn{3}{c}{ High-School Grades } \\
\cline { 2 - 3 } \cline { 7 - 8 } & English & Mathematics & Science & & English & Mathematics & Science \\
\hline Digit Symbol & $.25^{*}$ & .07 & .08 & & .19 & .19 & .08 \\
Space Code & -.07 & -.03 & -.05 & & -.07 & .18 & .01 \\
SPM-SF & $.28^{*}$ & .22 & .21 & & .08 & .27 & .20 \\
Space Matrix & $.35^{* *}$ & $.32^{*}$ & .23 & & .14 & $.34^{*}$ & .11 \\
\hline
\end{tabular}

aCorrelations for preparatory-school grades are Kendall's tau nonparametric correlations. $\quad{ }^{*} p<$ $.05 .{ }^{* *} p<.01$. 
and Gf measures. Although WM and Gf are distinct constructs, there is also a substantial amount of shared variance at the latent construct level (Ackerman et al., 2005; Engle et al., 1999; Fry \& Hale, 2000; McGrew, 2005; Unsworth \& Engle, 2005). In the absence of additional Gf measures, the Raven's tests, a measure of Gf, and WM measures loaded on the same factor in both of the present experiments. Space Matrix also had substantial relationships with the Raven's tests in both experiments (.53 and .44, respectively). Previous researchers have reported correlations of about $.30-.35$ between well-established WM measures (e.g., reading, counting, and operations span) and the Raven's tests (Conway et al., 2002; Engle et al., 1999; Unsworth \& Engle, 2005). Thus, Space Matrix would seem to compare quite favorably as a correlate of Gf, or even $g$, for those who argue that the Raven's tests are perhaps the best measures of general intelligence (Jensen, 1998; Prokosch et al., 2005). These results were further supported by comparisons of respective relationships with school grades.

Space Matrix and the Raven's tests had similar patterns of relationships with school subject grades in both samples, and the magnitudes of the correlations between the Raven's tests and school grades were similar to those reported by other researchers (Rushton, Skuy, \& Fridjhon, 2003). Although the present samples are too small to form the basis for any strong conclusions, it was encouraging to find that the correlations between school grades and Space Matrix were higher than those between the Raven's tests and every subject except high-school science. Interestingly, both tests had stronger relationships with preparatory English than with high-school English, which may reflect curriculum differences, assessment differences, or developmental trends. This is difficult to ascertain, however, without detailed knowledge of course components and assessment procedures. The significant relationships Space Matrix had with preparatory English and with both preparatory and high-school mathematics is consistent with research that has indicated that WM plays a role in reading and writing abilities (Floyd et al., 2003; Hitch, Towse, \& Hutton, 2001; Just \& Carpenter, 1992 ) and in mathematical abilities (Floyd et al., 2003; Hitch et al., 2001; Swanson \& Kim, 2007). In contrast, Space Code had no substantial relationships with any of the school subject grades. Digit Symbol, however, was significantly related to preparatory English and slightly less, albeit nonsignificantly, related to high-school English, a finding that is consistent with previous research that has indicated a relationship, at this age level, between Gs and basic reading skills (Floyd et al., 2003). On the whole, these results provide encouraging preliminary evidence for the external validity of Space Matrix but not of Space Code. Space Code will require further development before it can be used as an alternative measure of processing speed. Current versions of Space Code have been designed to reduce the visual and working memory demands of the task, in an effort to redress the observed relationships with these constructs.

There were a number of interesting findings in both experiments concerning the nonability measures. In Experi- ment 1 , there was a significant positive correlation between gender and Space Code, which indicated that males were quicker in this test. After controlling for the frequency of current and past computer gaming, however, the correlation was reduced almost to zero. Thus, it appeared that the relationship was mediated by levels of computer-gaming experience. Computer-gaming measures had substantial positive relationships with Space Code and Space Matrix, but they were similarly related to the WM/Gf composite. In Experiment 2, gender was not significantly correlated with either computer-game-like test, but current computer gaming was significantly correlated with Space Code scores. As in Experiment 1, however, this measure was also similarly related to WM/Gf composites. Thus, although it could be argued that computer-game-like tests are biased toward those who play more computer games, this would also seem to be true for the WM/Gf measures. Since the WM/Gf measures were also administered via computer, however, those who play more computer games may simply be more comfortable with computer administration of any test.

Future research might investigate whether this relationship holds for WM and Gf measures administered via paper-and-pencil or oral methods. If the relationship is not dependent on administration mode, a further possibility is that computer-game playing enhances attentional skills, visuospatial skills, or other skills associated with all of these tasks. Another possibility is that those with higher WM/Gf ability are more likely to enjoy playing computer games. Recent studies have begun to address some of these possibilities (Aliya, 2002; Castel, Pratt, \& Drummond, 2005; Enochsson et al., 2004; Quaiser-Pohl, Geiser, \& Lehmann, 2006), but this issue is clearly beyond the scope of the present study. It remains important to acknowledge that computer-game-like tests may not rank individuals in the same way that traditional tests do, however, even when a certain degree of construct validity is achieved. Future research will need to address this issue in greater detail.

A number of other issues will also need to be addressed. The present study involved small sample sizes and a limited range of ability levels and ages. Although the results thus far are encouraging - in particular, those for Space Matrix - research will need to be conducted using larger and more representative samples. Additionally, and perhaps more importantly, the range of tests used to assess construct validity needs to be expanded to represent a much larger range of cognitive abilities. In the absence of a wider range of tests, it is not possible to ascertain whether these computer-game-like tests are not also measuring other abilities.

Finally, another more pragmatic issue that arose highlights the many potentially interesting pitfalls that may lie ahead. In line with research that indicates that game players commonly adopt a trial-and-error approach when learning a new game (Miller, Schweingruber, \& Brandenburg, 2001; Mitchell \& Savill-Smith, 2004), some children in both the preparatory and high-school samples did not stop to read the instructions before beginning the actual test. Future versions of the computer-game-like tests should address this issue by incorporating more extensive 
trial-and-error practice. The challenge will be to maintain as much of the gaming experience as possible, with all of the potential advantages, while creating tools that are as reliable and valid as traditional measures.

\section{AUTHOR NOTE}

Correspondence concerning this article should be addressed to J. McPherson, School of Psychology, University of Adelaide, SA 5005, Australia (e-mail: jlmcpher@psychology.adelaide.edu.au).

\section{REFERENCES}

Ackerman, P. L., Beier, M. E., \& Boyle, M. O. (2002). Individual differences in working memory within a nomological network of cognitive and perceptual speed abilities. Journal of Experimental Psychology: General, 131, 567-589.

AcKerman, P. L., Beier, M. E., \& Boyle, M. O. (2005). Working memory and intelligence: The same or different constructs? Psychological Bulletin, 131, 30-60.

AliYA, K. S. (2002). The role of computer games in the development of theoretical analysis, flexibility and reflective thinking in children: A longitudinal study. International Journal of Psychophysiology, 45, 149.

Bors, D. A., \& Stokes, T. L. (1998). Raven's Advanced Progressive Matrices: Norms for first-year university students and the development of a short form. Educational \& Psychological Measurement, 58, 382-399.

Burns, N. R., \& NetTelbeck, T. (2003). Inspection time in the structure of cognitive abilities: Where does IT fit? Intelligence, 31, 237-255.

Carpenter, P. A., Just, M. A., \& Shell, P. (1990). What one intelligence test measures: A theoretical account of the processing in the Raven Progressive Matrices Test. Psychological Review, 97, 404-431.

Carroll, J. B. (1993). Human cognitive abilities: A survey of factoranalytic studies. Cambridge: Cambridge University Press.

Castel, A. D., Pratt, J., \& Drummond, E. (2005). The effects of action video game experience on the time course of inhibition of return and the efficiency of visual search. Acta Psychologica, 119, 217-230.

Cattell, R. B. (1966). The scree test for the number of factors. Multivariate Behavioral Research, 1, 245-276.

Colom, R., Abad, F. J., Rebollo, I., \& Shin, P. C. (2005). Memory span and general intelligence: A latent-variable approach. Intelligence, 33, 623-642.

Colom, R., \& SHIH, P. C. (2004). Is working memory fractionated onto different components of intelligence? A reply to Mackintosh and Bennett (2003). Intelligence, 32, 431-444.

Conway, A. R. A., Cowan, N., Bunting, M. F., Therriault, D. J., \& MinkofF, S. R. B. (2002). A latent variable analysis of working memory capacity, short-term memory capacity, processing speed, and general fluid intelligence. Intelligence, 30, 163-183.

Danthitr, V., Roberts, R. L., Schulze, R., \& Wilhelm, O. (2005). Mental speed: On frameworks, paradigms, and a platform for the future. In O. Wilhelm \& R. W. Engle (Eds.), Understanding and measuring intelligence (pp. 24-46). London: Sage.

Deary, I. J. (2001). Human intelligence differences: A recent history. Trends in Cognitive Sciences, 5, 127-130.

Engle, R. W., Tuholski, S. W., Laughlin, J. E., \& Conway, A. R. A. (1999). Working memory, short-term memory, and general fluid intelligence: A latent-variable approach. Journal of Experimental Psychology: General, 128, 309-331.

Enochsson, L., Isaksson, B., Tour, R., Kuellin, A., Hedman, L., Wredmark, T., \& Tsai-Felländer, L. (2004). Visuospatial skills and computer game experience influence the performance of virtual endoscopy. Journal of Gastrointestinal Surgery, 8, 876-882.

Fabrigar, L. R., Wegener, D. T., MacCallum, R. C., \& Strahan, E. J. (1999). Evaluating the use of exploratory factor analysis in psychological research. Psychological Methods, 4, 272-299.

FLOYD, R. G. (2005). Information-processing approaches to interpretation of contemporary intellectual assessment instruments. In D. P. Flanagan \& P. L. Harrison (Eds.), Contemporary intellectual assess- ment: Theories, tests, and issues (2nd ed., pp. 203-233). New York: Guilford.

Floyd, R. G., Shaver, R. B., \& McGrew, K. S. (2003). Interpretation of the Woodcock-Johnson III Tests of Cognitive Abilities: Acting on evidence. In F. A. Schrank \& D. P. Flanagan (Eds.), W-J III clinical use and interpretation: Scientist-practitioner perspectives (pp. 1-46). San Diego: Academic Press.

FrY, A. F., \& Hale, S. (1996). Processing speed, working memory, and fluid intelligence: Evidence for a developmental cascade. Psychological Science, 7, 237-241.

Fry, A. F., \& HaLe, S. (2000). Relationships among processing speed, working memory, and fluid intelligence in children. Biological Psychology, 54, 1-34.

Gathercole, S. E., \& Pickering, S. J. (2000). Working memory deficits in children with low achievements in the national curriculum at 7 years of age. British Journal of Educational Psychology, 70, 177-194.

Gentile, D. A., \& Walsh, D. A. (2002). A normative study of family media habits. Journal of Applied Developmental Psychology, 23, 157-178.

Henson, R. K., \& Roberts, J. K. (2006). Use of exploratory factor analysis in published research: Common errors and some comment on improved practice. Educational \& Psychological Measurement, 66, 393-416.

Hitch, G. J., Towse, J. N., \& Hutton, U. (2001). What limits children's working memory span? Theoretical accounts and applications for scholastic development. Journal of Experimental Psychology: General, 130, 184-198.

Horn, J. L., \& Blankson, N. (2005). Foundations for better understanding of cognitive abilities. In D. P. Flanagan \& P. L. Harrison (Eds.), Contemporary intellectual assessment: Theories, tests, and issues (2nd ed., pp. 41-68). New York: Guilford.

HorN, J. L., \& Noll, J. (1997). Human cognitive capabilities: Gf-Gc theory. In D. P. Flanagan, J. L. Genshaft, \& P. L. Harrison (Eds.), Contemporary intellectual assessment: Theories, tests and issues (pp. 5391). New York: Guilford.

Howell, D. C. (2007). Statistical methods for psychology (6th ed.). Belmont, CA: Thomson Wadsworth.

Jensen, A. R. (1982). Reaction time and psychometric G. In H. J. Eysenck (Ed.), A model for intelligence (pp. 93-132). New York: Springer.

JENSEN, A. R. (1998). The g factor: The science of mental ability. New York: Praeger.

Just, M. A., \& CARPENTER, P. A. (1992). A capacity theory of comprehension: Individual differences in working memory. Psychological Review, 99, 122-149.

Kane, M. J., Hambrick, D. Z., \& Conway, A. R. A. (2005). Working memory capacity and fluid intelligence are strongly related constructs: Comment on Ackerman, Beier, and Boyle (2005). Psychological Bulletin, 131, 66-71.

Kyllonen, P. C., \& Christal, R. E. (1990). Reasoning ability is (little more than) working-memory capacity?! Intelligence, 14, 389-433.

LaW, D. J., Morrin, K. A., \& Pellegrino, J. W. (1995). Training effects and working memory contributions to skill acquisition in a complex coordination task. Learning \& Individual Differences, 7, 207-234.

Lengenfelder, J., Bryant, D., Diamond, B. J., Kalmar, J. H., Moore, N. B., \& DeLucA, J. (2006). Processing speed interacts with working memory efficiency in multiple sclerosis. Archives of Clinical Neuropsychology, 21, 229-238.

Luo, D., Thompson, L. A., \& Detterman, D. K. (2006). The criterion validity of tasks of basic cognitive processes. Intelligence, $\mathbf{3 4}$, 79-120.

Lynn, R., Allik, J., \& IRwIng, P. (2004). Sex differences on three factors identified in Raven's Standard Progressive Matrices. Intelligence, 32, 411-424.

McGrew, K. (2005). The Cattell-Horn-Carroll theory of cognitive abilities. In D. P. Flanagan \& P. L. Harrison (Eds.), Contemporary intellectual assessment: Theories, tests, and issues (2nd ed., pp. 136180). New York: Guilford.

McGrew, K., \& Flanagan, D. P. (1998). The intelligence test desk reference (ITDR): Gf-Gc cross-battery assessment. Boston: Allyn \& Bacon.

McPherson, J., \& Burns, N. R. (2005). A speeded coding task using 
a computer-based mouse response. Behavior Research Methods, 37 , 538-544.

McPherson, J., \& Burns, N. R. (2007). Gs Invaders: Assessing a computer game-like test of processing speed. Behavior Research Methods, 39, 876-883.

Miller, L. M., Schweingruber, H., \& Brandenburg, C. L. (2001). Middle school students' technology practices and preferences. Journal of Educational Multimedia \& Hypermedia, 10, 125-140.

Mitchell, A., \& SAVIll-Smith, C. (2004). The use of computer and video games for learning: A review of the literature. London: Learning and Skills Development Agency.

MiYAKe, A. (2001). Individual differences in working memory: Introduction to the special section. Journal of Experimental Psychology: General, 130, 163-168.

Miyake, A., Friedman, N. P., Rettinger, D. A., Shah, P., \& HegARTY, M. (2001). How are visuospatial working memory, executive functioning, and spatial abilities related? A latent-variable analysis. Journal of Experimental Psychology: General, 130, 621-640.

Montanelli, R. G., \& Humphreys, L. G. (1976). Latent roots of random data correlation matrices with squared multiple correlations on the diagonal: A Monte Carlo study. Psychometrika, 41, 341-348.

Nyborg, H. (2003). The scientific study of general intelligence: A tribute to Arthur R. Jensen. Amsterdam: Pergamon.

Oberauer, K., Schulze, R., Wilhelm, O., \& Süß, H.-M. (2005). Working memory and intelligence - their correlation and their relation: Comment on Ackerman, Beier, and Boyle (2005). Psychological Bulletin, 131, 61-65.

O'CONNOR, B. P. (2000). SPSS and SAS programs for determining the number of components using parallel analysis and Velicer's MAP test. Behavior Research Methods, Instruments, \& Computers, 32, 396-402.

O'CONNOR, B. P. (2001). Extension: SAS, SPSS, and MATLAB programs for extension analysis. Applied Psychological Measurement, 25, 88.

Porter, D. B. (1995). Computer games: Paradigms of opportunity. Behavior Research Methods, Instruments, \& Computers, 27, 229-234.

Prokosch, M. D., Yeo, R. A., \& Miller, G. F. (2005). Intelligence tests with higher g-loadings show higher correlations with body symmetry: Evidence for a general fitness factor mediated by developmental stability. Intelligence, 33, 203-213.

Quaiser-Pohl, C., Geiser, C., \& Lehmann, W. (2006). The relationship between computer-game preference, gender, and mental-rotation ability. Personality \& Individual Differences, 40, 609-619.

Raven, J. C., Raven, J. E., \& Court, J. H. (1998). Progressive matrices. Oxford: Oxford Psychologists Press.

Rushton, J. P., Skuy, M., \& Fridjhon, P. (2003). Performance on Raven's Advanced Progressive Matrices by African, East Indian, and White engineering students in South Africa. Intelligence, 31, 123-137.

Schmid, J., \& LeIman, J. N. (1957). The development of hierarchical factor solutions. Psychometrika, 22, 53-61.
SCHWEIZER, K. (2005). An overview of research into the cognitive basis of intelligence. Journal of Individual Differences, 26, 43-51.

Schweizer, K., \& Moosbrugger, H. (2004). Attention and working memory as predictors of intelligence. Intelligence, 32, 329-347.

STANKov, L. (2000). Complexity, metacognition, and fluid intelligence. Intelligence, 28, 121-143.

Stankov, L., \& Roberts, R. D. (1997). Mental speed is not the "basic" process of intelligence. Personality \& Individual Differences, 22, 69-84.

Süß, H.-M., Oberauer, K., Wittmann, W. W., Wilhelm, O., \& Schulze, R. (2002). Working-memory capacity explains reasoning ability - and a little bit more. Intelligence, 30, 261-288.

SWANSON, L., \& KIM, K. (2007). Working memory, short-term memory, and naming speed as predictors of children's mathematical performance. Intelligence, 35, 151-168.

UNSWORTH, N., \& ENGLE, R. W. (2005). Working memory capacity and fluid abilities: Examining the correlation between Operation Span and Raven. Intelligence, 33, 67-81.

Velicer, W. F. (1976). Determining the number of components from the matrix of partial correlations. Psychometrika, 41, 321-327.

WAshburn, D. A. (2003). The games psychologists play (and the data they provide). Behavior Research Methods, Instruments, \& Computers, 35, 185-193.

WickelgRen, I. (1997). Working memory linked to intelligence. Science, 275, 1581-1582.

Wolff, H.-G., \& Preising, K. (2005). Exploring item and higher order factor structure with the Schmid-Leiman solution: Syntax codes for SPSS and SAS. Behavior Research Methods, 37, 48-58.

Wood, R. T. A., Griffiths, M. D., Chappell, D., \& Davies, M. N. O. (2004). The structural characteristics of video games: A psychostructural analysis. Cyberpsychology \& Behavior, 7, 1-10.

Woodcock, R. W., McGrew, K. S., \& Mather, N. (2001). The Woodcock-Johnson Test of Cognitive Abilities. Itasca, IL: Riverside.

YELLAND, N., \& LLOYD, M. (2001). Virtual kids of the 21st century: Understanding the children in schools today. Information Technology in Childhood Education Annual, 13, 175-192.

ZAJAC, I. T., \& Burns, N. R. (2007). Measuring auditory inspection time in primary school children. Journal of Individual Differences, 28, $45-53$

\section{NOTE}

1. Ability nomenclature and abbreviations are those used within Cattell-Horn-Carroll Theory (McGrew, 2005; McGrew \& Flanagan, 1998).

(Manuscript received March 29, 2007; revision accepted for publication March 12, 2008.) 